Research Paper

\title{
LncRNA SNHG5 can Regulate the Proliferation and Migration of Diffuse Large B Cell Lymphoma Progression via Targeting miR-181-5p/XIAP
}

\author{
Xiaojing Xing ${ }^{1,2}$, Tonghong $\mathrm{Xu}^{1,2}$, Bin $\mathrm{Liu}^{1,2}$, Qianxue Guo ${ }^{1,2}$ \\ 1. Department of Hematology and Breast Cancer, Cancer Hospital of China Medical University, Shenyang 110042, Liaoning, P.R.China. \\ 2. Department of Hematology and Breast Cancer, Liaoning Cancer Hospital \& Institute, Shenyang 110042, Liaoning, P.R.China.
}

$\square$ Corresponding author: Xiaojing Xing, Department of Hematology and Breast Cancer, Cancer Hospital of China Medical University, Liaoning Cancer Hospital and Institute, No. 44 Xiaoheyan Road, Dadong District, Shenyang 110042, Liaoning, P.R.China. E-mail: xxiaojing0225@163.com. ORCID ID: 0000-0002-1518-0871

(c) The author(s). This is an open access article distributed under the terms of the Creative Commons Attribution License (https://creativecommons.org/licenses/by/4.0/). See http://ivyspring.com/terms for full terms and conditions.

Received: 2021.03.16; Accepted: 2021.10.22; Published: 2022.01.01

\begin{abstract}
Background: It has been verified that long noncoding RNAs (IncRNAs) may participate in the pathogenesis of various human diseases. This study aims to investigate the roles of IncRNA SNHG5 in diffuse large B cell lymphoma (DLBC), especially the impacts of IncRNA SNHG5 on proliferation and migration of human diffuse large $B$ cell lymphoma cells and the related mechanism.

Methods: qRT-PCR analysis was carried out to examine the expression pattern of SNHG5 in DLBC tissue and adjacent normal tissue. The effect of SNHG5 on the proliferation, apoptosis, migration, and invasion of DLBC cells was detected by MTT, flow cytometry analysis, wound healing assay and transwell assay. The correlation between SNHG5, miR-181-5p and XIAP were certified by bioinformatics analysis, and dual-luciferase reporter assay. Furthermore, rescue assays were performed to analyze the effects of SNHG5-miR-181-5p-XIAP axis on the biological behaviors of diffuse large B cell lymphoma cells. Finally, the effects of SNHG5 axis on the growth of DLBC tumor was examined by in vivo analysis.

Results: SNHG5 was significantly upregulated in diffuse large B cell lymphoma tissues. Knockdown of SNHG5 inhibited the proliferation, migration, and invasion of diffuse large B cell lymphoma cells in vitro and in vivo. LncRNA SNHG5 acted as a ceRNA through binding with miR-181-5p in DLBC cells.

Conclusion: LncRNA SNHG5 may promote proliferation and migration of diffuse large $B$ cell lymphoma cells via targeting miR-181-5p/XIAP.
\end{abstract}

Key words: SNHG5, miR-181-5p, diffuse large B cell lymphoma, competing endogenous RNA

\section{Introduction}

Diffuse large B cell lymphoma (DLBC) one of the most diagnosed type of lymphoma worldwide [1-3]. Like many other type of cancers, the pathogenesis of DLBC is still unclear, and there is no specific treatment method for DLBC, leading to the poor prognosis and highmortality rate of the disease $[4,5]$. In clinical applications, the most important reason for the high mortality rate is the metastasis as well as recurrence of DLBC that occurred in most the cases [2, $3,6]$. Therefore, it is of great importance to further explore the underlying mechanism of DLBC and development new methods to improve the therapeutic efficacity as well as prognosis of the disease.

Long non-coding RNAs (LncRNAs) are a group of non-coding RNAs, and LncRNAs are often with the length of over 200 nucleotides, some of the LncRNAs can be kilobases in length $[7,8]$. With the in-depth investigation of the underlying mechanism LncRNAs, researchers found that LncRNAs can regulate various biological events, for example cell proliferation, migration, differentiation through affecting the gene expression at post-transcriptional level $[9,10]$. In the case of carcinogenesis, LncRNAs were found to be 
involved in the pathogenesis of many types of cancers, including DLBC $[9,11-14]$.

Based on the results of bioinformatic analysis using the data from TCGA database, it was found that LncRNA SNHG5 was one of the most significantly up-regulated LncRNAs in the DLBC; however, the specific underlying mechanism is still unknown. In the present study, we will explore the roles of LncRNA SNHG5 in DLBC and the possible mechanism. We hypothesized that SNHG5 may function as an onco-LncRNA in DLBC and knockdown of SNHG5 could inhibit both growth and migration of DLBC cells.

\section{Methods}

\section{Patients and clinical tissue samples}

A total number of 90 paired DLBC tumor samples and the adjacent normal tissues were obtained from DLBC patients at Department of Hematology and Breast Cancer, Cancer Hospital of China Medical University between Jan.2019 and Feb 2020. Serum samples of 90 healthy volunteers were also collected as the control group. The samples were stored immediately after surgery in liquid nitrogen until analyzed was performed. Patients were diagnosed with DLBC through mass biopsy or cytology, and all diagnosis meets the 2017 WHO diagnostic classification criteria for lymphoid tumors[3]. All patients are newly diagnosed, have not received relevant treatment before admission, and have no history of radiotherapy and/or chemotherapy. Exclude patients with secondary DLBC, autoimmune diseases and related histories of malignant tumors. Pathology reports of all patients were collected. The informed consent was obtained from each patient. This study was approved by the ethical committee of Cancer Hospital of China Medical University.

\section{Cell culture}

Human normal human B-lymphocytes GM12878 and human diffuse large B cell lymphoma cell lines SU-DHL-4, SU-DHL-6 and SU-DHL-10 were purchased from American Type Culture Collection (ADLBCC, USA). The DLBC cells were cultured in the RPMI-1640 Medium (Invitrogen, Carlsbad, CA, USA). Medium was supplemented with $10 \%$ fetal bovine serum (FBS, Fisher, NY, USA) and 100 units $/ \mathrm{ml}$ penicillin and $100 \mu \mathrm{g} / \mathrm{ml}$ streptomycin at $37^{\circ} \mathrm{C}$ with $5 \% \mathrm{CO}_{2}$ and $95 \%$ humidity.

\section{Transfection}

SNHG5 over-expression plasmid was synthesized by Shanghai GenePharma Co.,Ltd (Shanghai, China). SU-DHL-4 and SU-DHL-6 cells were transfected with SNHG5 shRNA by lipofectamine 3000 (Invitrogen, Carlsbad, CA, USA) in accordance with the manufacturer's instructions. The transfection efficiency was determined by RT-qPCR method.

\section{RT-qPCR}

The miRNeasy Mini kit (Qiagen, Valencia, USA) was used to extract total RNA from tissues and cells in accordance with the manufacturer's instructions. The concentration and quality of the RNAs were determined by NanoDrop 2000 (Thermo Fisher, Wilmington, USA). The first-strand cDNA was synthesized by TransScript first-strand cDNA synthesis supermix (Transgen, Beijing, China) in accordance with the manufacturer's instructions. RT-qPCR assay was performed using the SYBR green qPCR supermix (Applied Biosystems Life Technologies, Foster, USA) in ABI prism 7500 sequence detection system (Applied Biosystems Life Technologies, Foster, USA). Conditions of the PCR reactions were presented as below: $55^{\circ} \mathrm{C}$ for $10 \mathrm{~min}$, 40 cycles of $95^{\circ} \mathrm{C}$ for $30 \mathrm{~s}, 55-59^{\circ} \mathrm{C} 30 \mathrm{~s}$ and $72{ }^{\circ} \mathrm{C}$ for $42 \mathrm{~s}$. Fold changes of the target genes was calculated by $2^{-\Delta \Delta C t}$ methods (cycle threshold), and expression levels of miRNA and lncRNA/target gene were normalized by U6 and GADPH, respectively.

\section{Cell proliferation assay}

The 5-diphenyltetrazolium bromide (MTT) assay was adopted to examine the proliferation of the DLBC cells in different groups. Briefly, cells were seeded on 96-well plates $\left(5 \times 10^{3} /\right.$ well $)$ and incubated with 100 pl $0.5 \mathrm{mg} / \mathrm{ml} \mathrm{MTT}$ for $4 \mathrm{~h}$ in an incubator, and precipitate was dissolved in $150 \mu \mathrm{l}$ dimethylsulfoxide (DMSO). The optical density value of each well at 570 $\mathrm{nm}$ was evaluated after shaking for $10 \mathrm{~min}$.

\section{Flow cytometry assay}

At $48 \mathrm{~h}$ after transfection, the cells of different groups were collected and mixed with $5 \mu \mathrm{l}$ Annexin-V-fluorescein isothiocyanate (FIDLBC) and $2.5 \mu \mathrm{l}$ propidium iodide (PI). The apoptosis of the cells was determined by a FACSAria Sorter (Becton Dickinson, San Jose, USA).

\section{Scratch wound healing assay}

DLBC cells were growed to $100 \%$ confluence in 6-well plates and then scratched by a $10 \mu \mathrm{ltip}$. After that, cells were incubated at the temperature of $37^{\circ} \mathrm{C}$ with $5 \% \mathrm{CO}_{2}$ and $95 \%$ humidified atmosphere for 24 $\mathrm{h}$. The migration areas at $0 \mathrm{~h}$ and $24 \mathrm{~h}$ were imaged with an inverted microscope and analyzed by Image J.

\section{Transwell assay}

Transwell cell invasion assay was carried out in 
24-well plates using the transwell chambers (Corning Inc., Corning, USA) fitted by a $8 \mu \mathrm{m}$ pores polyethylene terephthalate filter membrane. Cells $(5 \times$ $10^{4}$ ) were placed on the the upper chamber, and the lower chamber was filled with medium containing $10 \%$ FBS. After incubating for $24 \mathrm{~h}$, cells traversed to reverse face of the membrane were stained with crystal violet and imaged. The images were captured from 5 randomly chosen fields by a microscope.

\section{Western blotting}

The antibodies were all purchased from Santa Cruz Biotechnology (Dallas, USA). Samples (15 $\mu \mathrm{g}$ protein/lane) were electrophoresed by SDS-PAGE method and transferred onto PVDF membranes by iBlot Gel Transfer Device (Thermo Fisher). The membranes were then blocked by Blocking One (Nacalai Tesque, Kyoto, Japan) and incubated with the primary antibodies at $4{ }^{\circ} \mathrm{C}$ overnight. On day 2, the protein bands were incubated with horseradish peroxidase (HRP)-conjugated secondary antibodies (Cell Signaling Technology), and treated with ECL Prime Western Blotting Detection Reagents (GE Healthcare Life Sciences, Little Chalfont, UK). Finally, ImageQuant TL GE Healthcare Life Sciences) system was applied to digitize the strength of the band.

\section{Luciferase activity assay}

SNHG5 cDNA and miR-181-5p fragments including microRNA binding sites was cloned into the pmirGLO plasmids (Promega, Madison, USA). Mutant SNHG5 (pmirGLO-SNHG5-MUT) and Mutant miR-181-5p (pmirGLO-miR-181-5p-MUT) were also generated as the control. Luciferase reporter plasmid and miR-181-5p mimics, XIAP mimics or miR-NC mimics were co-transfected into 293 cells by Lipofectamine 2000. $48 \mathrm{~h}$ after transfection, the relative luciferase activity was examined by luminometer by Dual-Luciferase Reporter Assay System (Promega).

\section{Xenograft nude-mice tumor models}

20 Male nude mice (Balb/c-nu/nu, 4-6 weeks) were purchased from the animal center of Nanjing Medical University (Nanjing, China) and kept in specific pathogen-free (SPF) room supplied with food and water adlibitum. DLBC cells were subcutaneously injected into the right flank of the mice to create the xenograft tumor. 28 days later, the mice were sacrificed and the weight of the tumors was recorded.

\section{Statistical analysis}

Data were expressed as mean \pm SD. The comparisons between two groups or multiple groups were examined by Student's t-test or one-way analysis of variance, respectively. $\mathrm{P}<0.05$ was considered as statistical significance. The statistical analysis in this study were carried out by SPSS version 13.0 (Chicago, IL, USA).

\section{Results}

\section{Upregulation of SNHG5 in DLBC serum samples and cell lines}

First of all, the expression of SNHG5 in DLBC tumor samples and normal tissues were compared. Figure 1 shows the results of bioinformatic analysis. We found that the expression of SNHG5 markedly increased in DLBC tumor samples compared with the normal tissue (Figure 1A, p<0.01); moreover, we collected 90 DLBC samples and adjacent normal tissues (Table 1), and the expression level of SNHG5 were compared. We found that SNHG5 were significantly increased in DLBC tumor samples compared with the normal tissue (Figure 1B, $\mathrm{p}<0.01$ ). Furthermore, the expression of SNHG5 in DLBC cell lines and normal human B-lymphocytes were also compared. The levels of SNHG5 were increased in all DLBC cell lines (Figure 1C, p<0.05), especially SU-DHL-4, thus, it has been used for the in vitro studies.

Table 1. Associations between the expression level of SNHG5 and clinical characteristics of DLBCL patients $(n=90)$.

\begin{tabular}{llll}
\hline Characteristic & \multicolumn{2}{l}{$\mathrm{N}(\%)$} & P value \\
\cline { 2 - 3 } & Low (40) & High (50) & 0.3961 \\
\hline Sex & 22 & 23 & \\
male & 18 & 27 & 0.4985 \\
female & & & \\
Age & 26 & 29 & \\
$\leq 60$ & 14 & 21 & 0.0339 \\
60 & & & \\
Ann Arbor stages & 25 & 20 & \\
I-II & 15 & 30 & \\
III-IV & & & \\
ECOG & 24 & 26 & 0.4479 \\
$0-1$ & 16 & 24 & \\
$\geq 2$ & & & \\
IPI score & 28 & 19 & 0.2076 \\
$0-2$ & 12 & 31 & \\
$3-5$ & & & \\
LDH ratio & 21 & 18 & \\
$\leq 1$ & 19 & & \\
$>1$ & & &
\end{tabular}

\section{Knockdown of SNHG5 inhibits the proliferation and promotes the apoptosis of DLBC cells}

To investigate the roles of SNHG5 in regulating the proliferation and apoptosis of DLBC cells, DLBC cells was treated with SNHG5 shRNA, and the proliferation and apoptosis of the cells were determined. We found that SNHG5 over-expression plasmid dramatically inhibited the proliferation and 
promoted the apoptosis of DLBC cells in vitro (Figure $2 \mathrm{~A}$ and $\mathrm{C}, \mathrm{p}<0.01)$. Moreover, the expression of anti-apoptotic protein Bcl-2 was decreased and the expression of pro-apoptotic protein BAX, Caspase-3 were increased by SNHG5 shRNA (Figure 2B, $\mathrm{p}<0.01)$.

\section{Knockdown of SNHG5 inhibits the migration and invasion of DLBC cells in vitro}

Next, the roles of SNHG5 in regulating the migration and invasion of DLBC cells were also explored. It has been observed that SNHG5 shRNA significantly inhibited the migration (Figure $3 \mathrm{~A}$ and $C, p<0.01$ ) and invasion (Figure $3 B$ and D, $p<0.01$ ) of DLBC cells in vitro. The expression of migration and invasion related proteins MMP-2 and MMP-9 was decreased by over-expression of SNHG5 (Figure 3E, $\mathrm{p}<0.01)$.

\section{Knockdown of SNHG5 inhibits the growth of the DLBC tumor in vivo}

Next, to the in vivo roles of SNHG5 in DLBC was also explored by the xenograft mice tumor models. We found that the weight of the DLBC tumors was significantly lower in SNHG5 knockdown group in comparison with the controls (Figure 4A and B, $p<0.01$ ), suggesting that knockdown of SNHG5 can

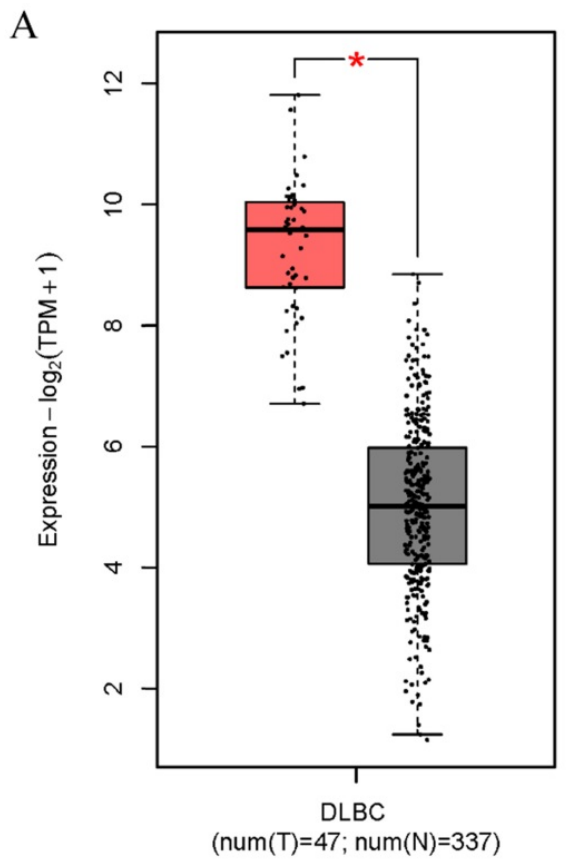

inhibit the growth of the tumor in vivo.

\section{SNHG5 inhibits the proliferation and migration of DLBC cells via targeting miR-181-5p/XIAP}

The underlying mechanism of SNHG5 as an onco-LncRNA in DLBC was explored. First, Using bioinformatics methods to predict the SNHG5/miR181-5p/XIAP axis regulatory network in DLBC cells (Figure 5A and Figure 6A). miR-181-5p was identified as a target of SNHG5, and XIAP was identified as a target of miR-181-5p. Next, we found miR-181-5p was significantly decreased in DLBC tissue, and the expression level of SNHG5 and miR-181-5p in DLBC tissue was negatively correlated with (Figure $5 \mathrm{~B}$ and C). Moreover, results of dual-luciferase reporter assay confirmed the targeting relationship between SNHG5 and miR-181-5p, miR-181-5p and XIAP. (Figure 5D, Figure $6 \mathrm{~B}, \mathrm{C}$ and D). Finally, we also found that co-transfection of SNHG5 over-expression plasmid and miR-181-5p mimics partially abrogated the anti-tumor effects of SNHG5 shRNA by promoting the proliferation (Figure 7A), migration (Figure 7C), invasion (Figure 7D), and inhibiting the apoptosis (Figure 7B) of DLBC cells.

B

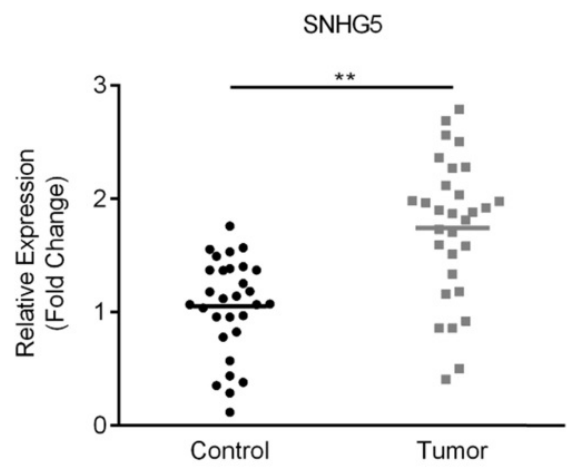

$\mathrm{C}$

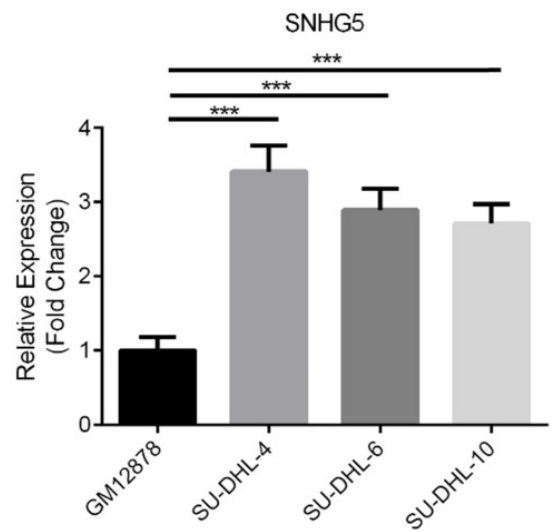

Figure 1. Upregulation of SNHG5 in DLBC serum samples and cell lines. (A) Results of bioinformatic analysis. (B) Comparison of the expression of SNHG5 in DLBC tumor samples and the adjacent normal tissues. (C) The expression of SNHG5 in DLBC cell lines and normal human B-lymphocytes. ${ }^{* *} \mathrm{p}<0.01,{ }^{* * *} \mathrm{p}<0.001$. 
A

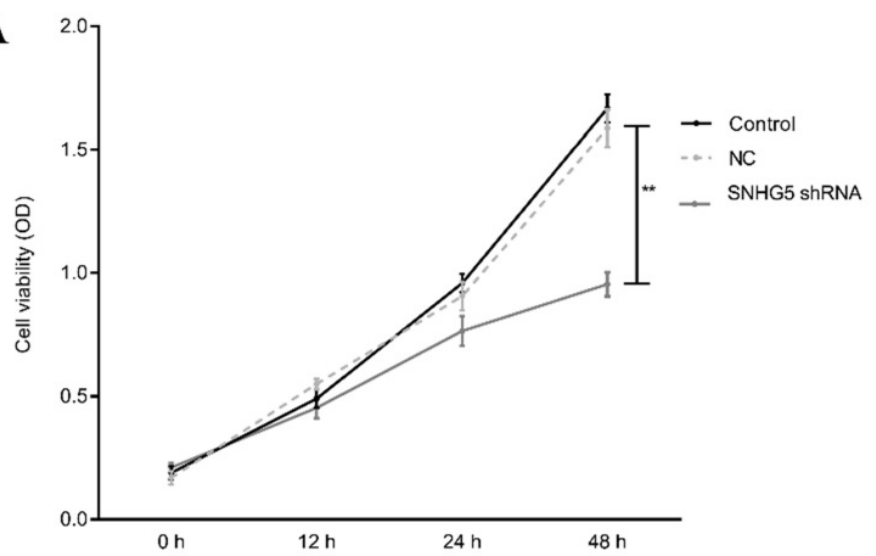

B

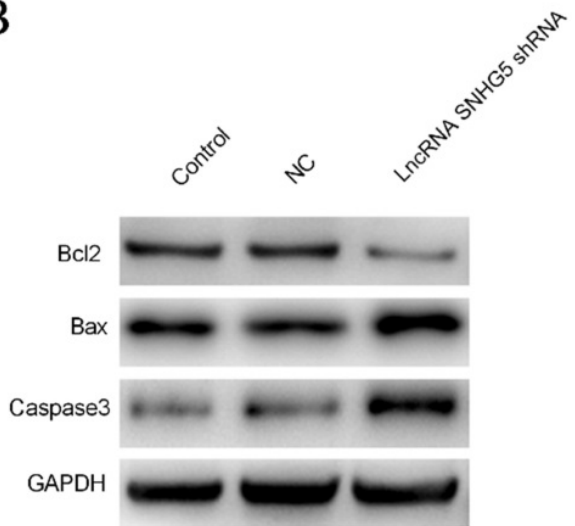

$\mathrm{C}$

Control

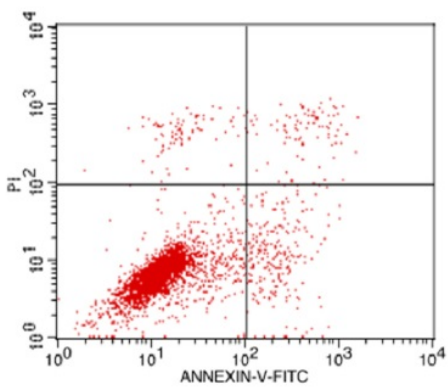

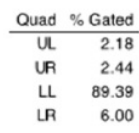

Figure 2. Knockdown of SNHG5 inhibits the proliferation and promotes the apoptosis of DLBC cells. (A) Cell proliferation by MTT assay. (B) Expressions of proliferation and apoptosis related proteins. (C)Cell apoptosis by flow cytometry assay. ${ }^{* *} \mathrm{p}<0.01,{ }^{* * *} \mathrm{p}<0.001$.

A

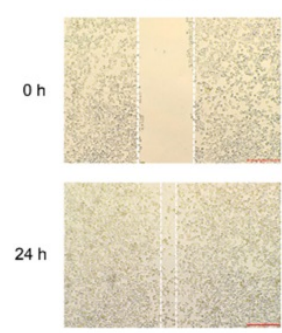

B

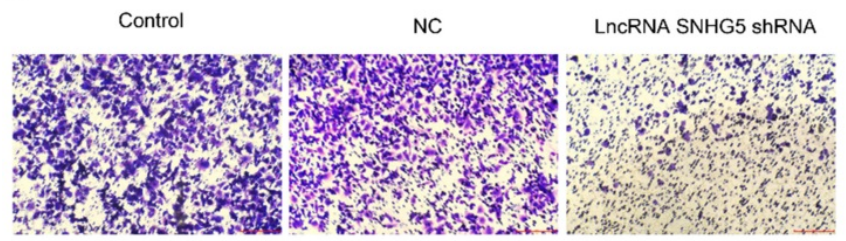

D

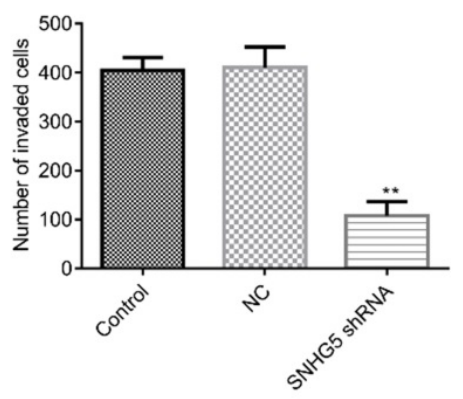

E

SNHG5 shRNA

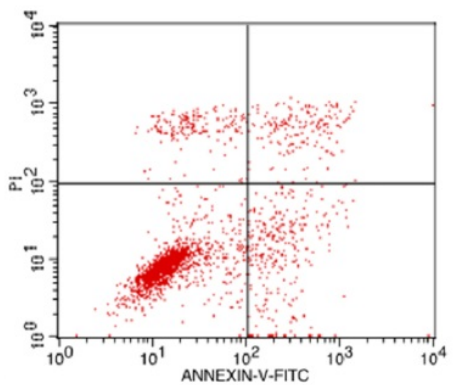

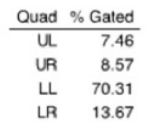

C

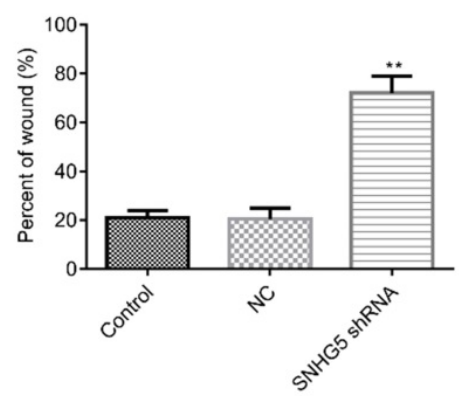
invasion ability by transwell assay. (E) Expressions of migration and invasion related proteins. ${ }^{* *} \mathrm{p}<0.01, * * * \mathrm{p}<0.001$. 


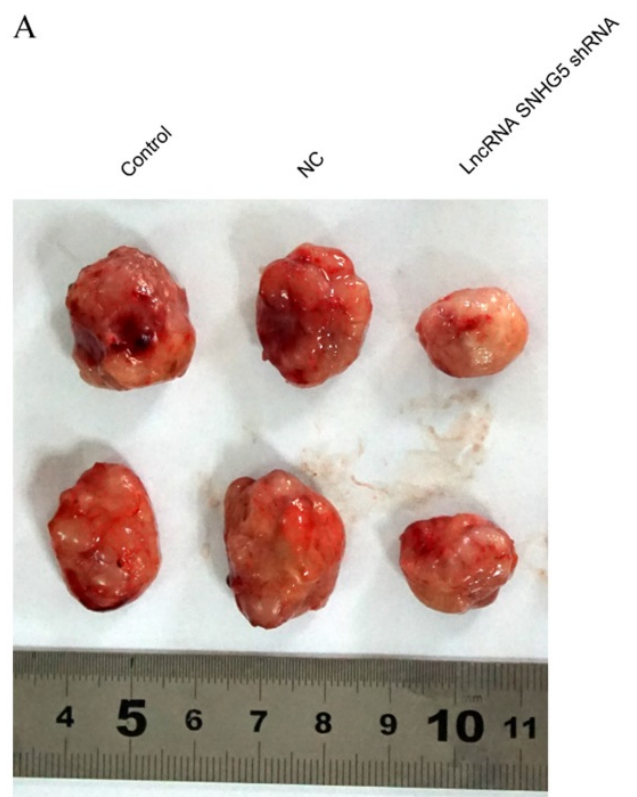

$\mathrm{B}$

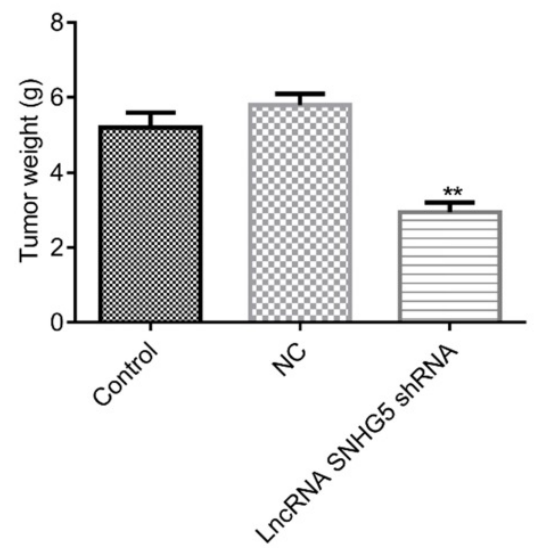

Figure 4. Knockdown of SNHG5 inhibits the growth of the DLBC tumor in vivo. (A and B) Weight of the tumors in different groups. $* * p<0.01, * * * p<0.001$.

A

SNHG5

$$
\begin{array}{ll}
\text { A AGUA C } \\
\text { CG } \\
\text { G CAGUG } \\
\text { GU } & \text { I } 111.1 \\
\text { GU GUCGC }
\end{array}
$$

hsa-miR-181a-5p
$\mathrm{B}$

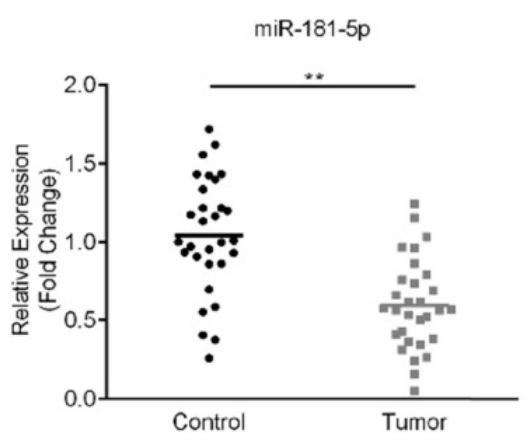

$\mathrm{D}$

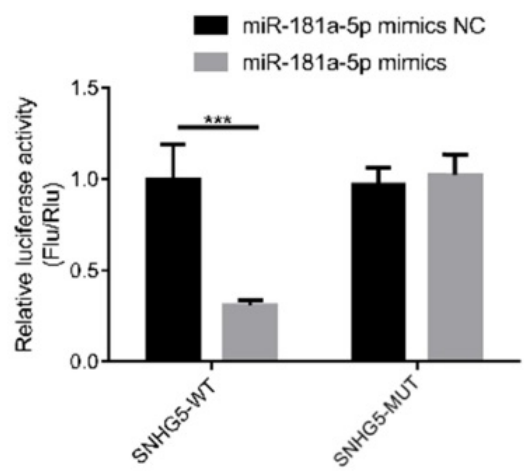

Figure 5. miR-181-5p is a target of SNHG5. (A) miR-181-5p was identified as a target of SNHG5 by bioinformatic method. (B) Expression of miR-181-5p in DLBC tissue and the adjacent normal tissue. (C) Correlation between the levels of SNHG5 and miR-181-5p in DLBC tissues. (D) Results of dual-luciferase reporter assay. $* * p<0.01$, $* * * *<<0.001$.

\section{Discussion}

In the present study, the roles of LncRNA SNHG5 in DLBC and the underlying mechanism were explored. We found that LncRNA SNHG5 may serve as an onco-LncRNA by inhibit the proliferation and migration of DLBC cells via modulating miR-181-5p/XIAP expression. 
A

XIAP 5’ UCUUAUUACCCUGAUUG AAUGUG

IIIIIII

miR-181-5p 3' UGAGUGGCUGUCGCAACUUACAA
B

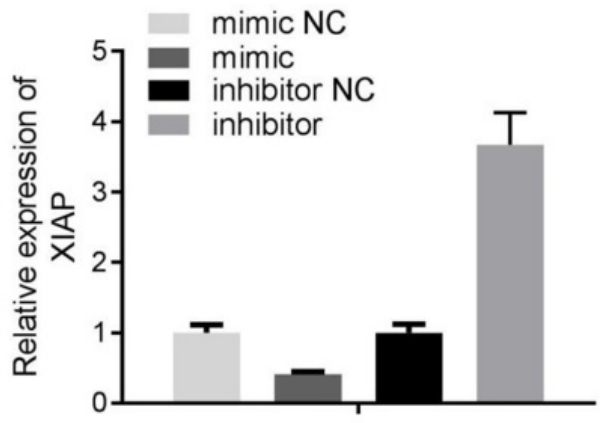

D

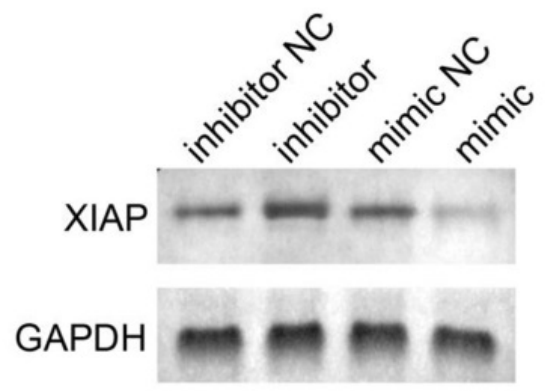

C

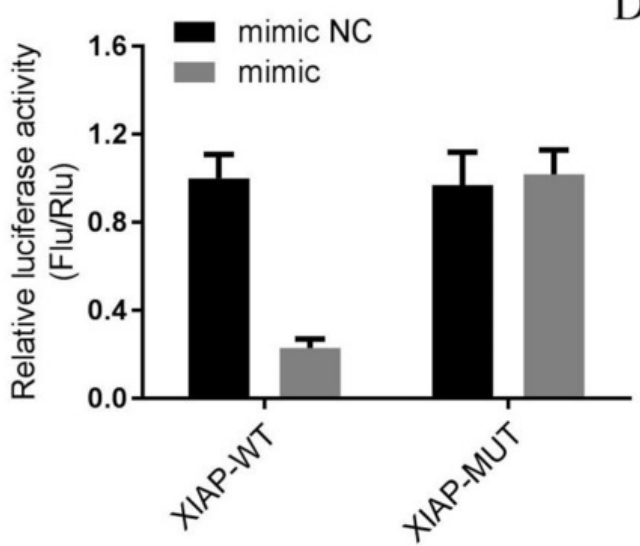

Figure 6. XIAP is a target of miR-181-5p. (A) XIAP was identified as a target of miR-181-5p by bioinformatic method. (B) The expression level of miR-181-5p was determined by qPCR in 4 DLBC lines (mimic NC, mimic, inhibitor NC and inhibitor). (C) Results of dual-luciferase reporter assay. (D) The expression level of miR-181-5p was determined by Western bolt in 4 DLBC lines (mimic NC, mimic, inhibitor NC and inhibitor).

A

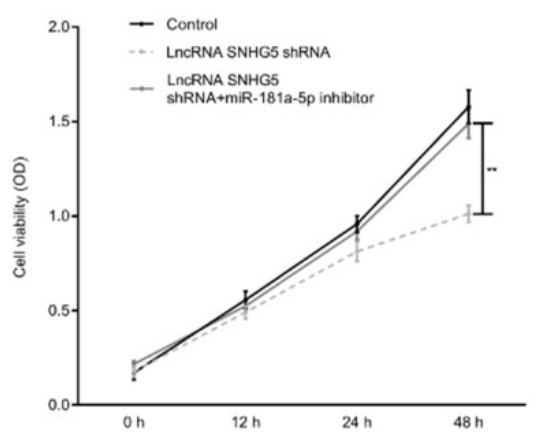

B

Control

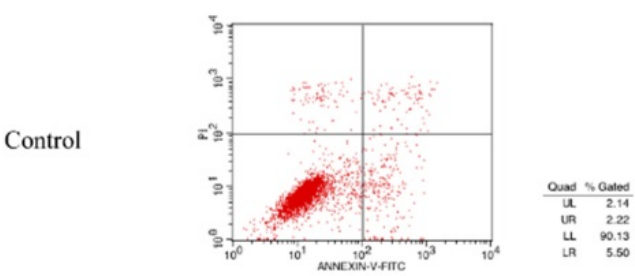

LncRNA SNHG5 ShRNA
$\mathrm{C}$

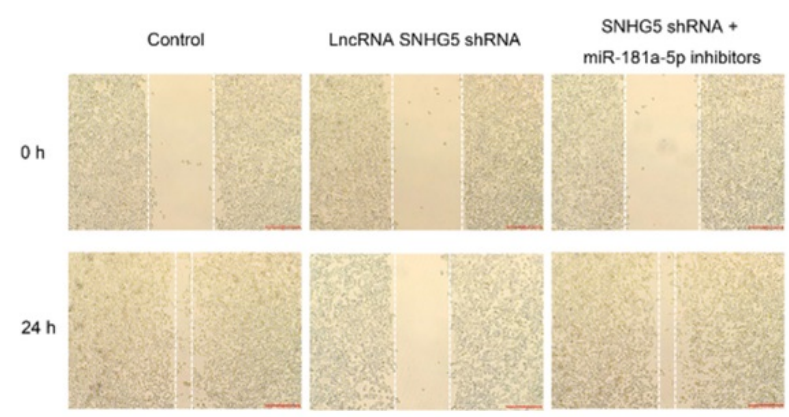

D

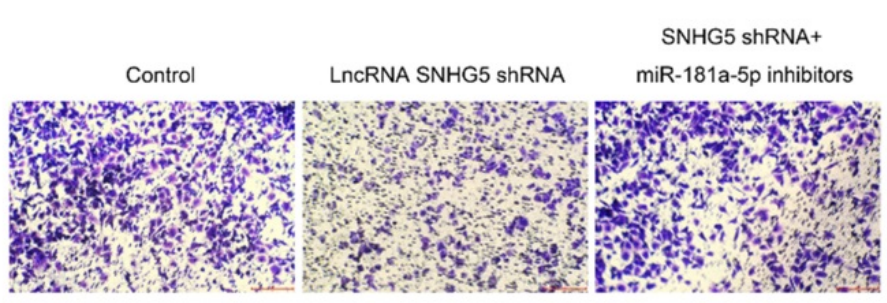

Figure 7. SNHG5 inhibits the proliferation and migration of DLBC cells via targeting miR-181-5p. (A) Cell proliferation by MTT assay. (B) Cell apoptosis by flow cytometry assay. (C) Cell migration ability by wound healing assay. (D) Cell invasion ability by transwell assay $* * p<0.01, * * * p<0.001$. 
The roles of SNHG5 in different type of cancers have been discussed previously. For example, upregulation of SNHG5 may induce the angiogenesis in acute myelogenous leukemia [15]; On the other hand, SNHG5 can regulate miR-205-5p expression and contributes to development of renal cell carcinoma[16]; furthermore, SNHG5 may function as an oncogenic LncRNA in nasopharyngeal carcinoma via targeting miR-1179 [17]. In DLBC, the roles of SNHG5 have not yet been discussed. Interestingly, results of bioinformatic analysis based on the data from the TCGA databased suggested that SNHG5 is one of the most significantly up-regulated LncRNAs in DLBC, suggesting that SNHG5 may also play carcinogenic roles in DLBC. In this study, we observed the decreased expression of SNHG5 in DLBC tumor tissue and cell lines, which was consistent with the results of the bioinformatic analysis of the TCGA databased. The above results proved that SNHG5 was over-expressed in DLBC and may sever as an onco-LncRNA.

Like any other type of cancers, the un-controlled growth and metathetic ability of DLBC lead to the high recurrent and mortality rate of the disease [18-20]. Therefore, an effective molecular therapeutic target of DLBC should be a key regulator that affect the proliferation and migration of the DLBC cells. In the present study, we found that knockdown of SNHG5 significantly suppressed the carcinogenic behavior of DLBC cells by inhibiting the proliferation, migration, invasion and promoting the apoptosis of DLBC cells in vitro and in vivo. These data suggested that SNHG5 may serve as potential novel therapeutic for the targeted-therapy for DLBC.

In previous studies, the interaction between LncRNAs and miRNAs were known as the most commonly underlying mechanism for LncRNAs to exert their biological functions via "sponging" and silencing the target miRNAs [11, 21-23]. With the development of the bioinformatics, the target miRNAs of LncRNAs can be predicted by virous methods. We used online bioinformatic tool starbased 3.0, and miR-181-5p has been identified as a target miRNA of SNHG5. By literate research, we found that miR-181-5p function as a tumor suppressor in different type of cancers [24-26]. Therefore, we hypothesized that LncRNA SNHG5 may exert its oncogenic function via down-regulating the expression of miR-181-5p. To verify this hypothesis, we performed a series of analysis and confirmed the negative correlation relationship between the levels of miR-181-5p and SNHG5 in DLBC tumor tissue; moreover, the direct targeting relationship between SNHG5 and miR-181-5p was also confirmed by dual luciferase reporter assay. Interestingly, miR-181-5p mimics partially abrogated the anti-tumor effects of SNHG5 shRNA in DLBC cells, by promoting the proliferation, migration, invasion, and inhibiting the apoptosis of the cells in vitro. In order to further determine the regulatory effect of SNHG5 on DLBC cells, we analyzed the LncRNAs/miRNAs/mRNA network of SNHG5 in DLBC cells. Discover the target gene XIAP of miR-181-5p through bioinformatics analysis and dual luciferase reporter gene detection. The study by Gu et al. has shown that the abnormal expression of XIAP is related to the apoptosis of DLBC cells [27], which is similar to the regulation mechanism of SNHG5. Taken together, we speculate that there is an Lnc SNHG5/ miR-181-5p / XIAP axis in DLBC cells, and SNHG5 can serves as onco-LncRNA to regulate the development of DLBC, which in turn promote the proliferation and migration of the cancer cells.

\section{Conclusion}

To sum up, we reported for the first time that LncRNA SNHG5 may function as a ceRNA to promote proliferation, migration, and invasion of diffuse large $B$ cell lymphoma cells through modulating miR-181-5p expression. Our data suggested that targeting the SNHG5/miR-181-5p/ XIAP axis may be a potential method for the treatment of DLBC.

\section{Acknowledgements}

The present study was funded by the Foundation of Department of Science and Technology, Liaoning Province (No. 2015020254) and the $\mathrm{Wu}$ Jieping Medical Foundation (No. 320.6750.17250).

\section{Competing Interests}

The authors have declared that no competing interest exists.

\section{References}

1. Salles G, Dlugosz-Danecka M, Ghesquieres H and Jurczak W. Tafasitamab for the treatment of relapsed or refractory diffuse large B-cell lymphoma. Expert Opin Biol Ther. 2021; 21(4): 455-463

2. Caeser R, Walker I, Gao J, et al. Acquired CARD11 mutation promotes BCR independence in Diffuse Large B Cell Lymphoma. JCO Precis Oncol. 2021; 5: 145-152

3. Choi M, O'Malley P. Diagnostically relevant updates to the 2017 WHO classification of lymphoid neoplasms. Annals of Diagnostic Pathology, 2018; 37(1): 67

4. Zhu L, Meng Y, Guo L, et al. Predictive value of baseline (18)F-FDG PET/CT and interim treatment response for the prognosis of patients with diffuse large B-cell lymphoma receiving R-CHOP chemotherapy. Oncol Lett. 2021; 21(2): 132

5. Lucioni M, Pescia C, Bonometti A, et al. Double expressor and double/triple hit status among primary cutaneous diffuse large B cell lymphoma: A comparison between leg type and NOS Subtypes. Hum Pathol. 2021; 111: 1-9

6. Morschhauser F, Feugier P, Flinn IW, et al. A phase 2 study of venetoclax plus $\mathrm{R}-\mathrm{CHOP}$ as first-line treatment for patients with diffuse large B-cell lymphoma. Blood. 2021; 137(5): 600-609 
7. Meng H, Zhao B and Wang Y. FOXM1-induced upregulation of lncRNA OR3A4 promotes the progression of diffuse large B-cell lymphoma via Wnt/beta-catenin signaling pathway. Exp Mol Pathol. 2020; 115: 104451

8. Qian CS, Li LJ, Huang HW, Yang HF and Wu DP. MYC-regulated lncRNA NEAT1 promotes $B$ cell proliferation and lymphomagenesis via the miR-34b-5p-GLI1 pathway in diffuse large B-cell lymphoma. Cancer Cell Int. 2020; 20(1): 1-13

9. Shi X, Cui Z, Liu X, et al. LncRNA FIRRE is activated by MYC and promotes the development of diffuse large B-cell lymphoma via Wnt/beta-catenin signaling pathway. Biochem Biophys Res Commun. 2019; 510(4): 594-600

10. Su Y, Sun B, Lin $X$, et al. Therapeutic strategy with artificially-designed i-lncRNA targeting multiple oncogenic microRNAs exhibits effective antitumor activity in diffuse large B-cell lymphoma. Oncotarget. 2016; 7(31): 49143-49155

11. Wang QM, Lian GY, Song Y, Huang YF and Gong Y. LncRNA MALAT1 promotes tumorigenesis and immune escape of diffuse large $B$ cell lymphoma by sponging miR-195. Life Sci. 2019; 231: 116335

12. Sun YJ, Li J and Chen CH. Effects of miR-221 on the apoptosis of non-small cell lung cancer cells by IncRNA HOTAIR. Eur Rev Med Pharmacol Sci. 2019; 23(10): 4226-4233

13. Chen $\mathrm{W}$, Hang $\mathrm{Y}, \mathrm{Xu} \mathrm{W}$, et al. BLACAT1 predicts poor prognosis and serves as oncogenic lncRNA in small-cell lung cancer. J Cell Biochem. 2019; 120(2): 2540-2546

14. Zhang L, Kang W, Lu X, Ma S, Dong L and Zou B. LncRNA CASC11 promoted gastric cancer cell proliferation, migration and invasion in vitro by regulating cell cycle pathway. Cell Cycle. 2018; 17(15): 1886-1900.

15. Li ZJ, Cheng J, Song Y, Li HH and Zheng JF. LncRNA SNHG5 upregulation induced by YY1 contributes to angiogenesis via miR-26b/CTGF/VEGFA axis in acute myelogenous leukemia. Lab Invest. 2021; 101(3): 341-352.

16. Xiang W, Lv L, Zhou G, et al. The IncRNA SNHG5-mediated miR-205-5p downregulation contributes to the progression of clear cell renal cell carcinoma by targeting ZEB1. Cancer Med. 2020; 9(12): 4251-4264.

17. Liu D, Wang Y, Zhao Y and Gu X. LncRNA SNHG5 promotes nasopharyngeal carcinoma progression by regulating miR-1179/HMGB3 axis. BMC Cancer. 2020; 20(1): 1-11.

18. Fu DW and Liu AC. LncRNA SBF2-AS1 Promotes Diffuse Large B-Cell Lymphoma Growth by Regulating FGFR2 via Sponging miR-494-3p. Cancer Manag Res. 2021; 13: 571-578.

19. Gao Q, Li Z, Meng L, Ma J, Xi Y and Wang T. Transcriptome profiling reveals an integrated mRNA-lncRNA signature with predictive value for long-term survival in diffuse large B-cell lymphoma. Aging (Albany NY). 2020; 12(22): 23275-23295.

20. Zhao L, Liu Y, Zhang J, Liu $Y$ and Qi Q. LncRNA SNHG14/miR-5590-3p/ZEB1 positive feedback loop promoted diffuse large B cell lymphoma progression and immune evasion through regulating PD-1/PD-L1 checkpoint. Cell Death Dis. 2019; 10(10): 713-731.

21. Zhong Y, Wang J, Lv W, Xu J, Mei S and Shan A. LncRNA TTN-AS1 drives invasion and migration of lung adenocarcinoma cells via modulation of miR-4677-3p/ZEB1 axis. J Cell Biochem. 2019; 120(10): 17131-17141.

22. Zhao $\mathrm{H}, \mathrm{Yu} \mathrm{H}$, Zheng J, et al. Lowly-expressed IncRNA GAS5 facilitates progression of ovarian cancer through targeting miR-196-5p and thereby regulating HOXA5. Gynecol Oncol. 2018; 151(2): 345-355.

23. He B, Bai Y, Kang W, Zhang X and Jiang X. LncRNA SNHG5 regulates imatinib resistance in chronic myeloid leukemia via acting as a CeRNA against MiR-205-5p. Am J Cancer Res. 2017; 7(8): 1704-1713.

24. Ma XF, Qin J and Guo XH. MiR-181-5p protects mice from sepsis via repressing HMGB1 in an experimental model. Eur Rev Med Pharmacol Sci. 2020; 24(18): 9712-9720.

25. Wang YL, An XH, Zhang XQ, Liu JH, Wang JW and Yang ZY. Morphine induces the apoptosis of mouse hippocampal neurons HT-22 through upregulating miR-181-5p. Eur Rev Med Pharmacol Sci. 2020; 24(12): 7114-7121.

26. Cui Y, Yin K, Gong Y, Qu Y, Liu H and Lin H. Atrazine induces necroptosis by miR-181-5p targeting inflammation and glycometabolism in carp lymphocytes. Fish Shellfish Immunol. 2019; 94: 730-738.

27. Gu J, Thompson J, Mavis $\mathrm{C}$, et al. Targeting MDM2 and XIAP By Idasanutlin in Diffuse Large B-Cell Lymphoma. Blood. 2019; 134: 5301. 